\title{
Selection of Conveyer Belt Goods-picking Method Based on Working Hour*
}

\author{
Shengjie Ke \\ Fuzhou University of International Studies and Trade \\ Fuzhou, China 350202
}

\begin{abstract}
In order to make clear the principle and effect of distribution center's using of conveyer belt to reduce working hour and to discuss the decision-making step to lead in conveyer belt for auxiliary goods-picking, in this research, the model for calculating goods-picking working hour by virtue of order data is established, and the difference in working hour constitution whether the conveyer belt is used for goods-picking when order data scale is different is analyzed. The result shows that, when the conveyer belt is used, the increase of moving time of goodspicking operator along with the increase of order data scale can be avoided, thus the working hour is reduced; the distribution center can determine whether the conveyer belt for goodspicking should be used by comparing salary cost converted by the reduced working hour and the maintenance cost for conveyer belt lead-in.
\end{abstract}

Keywords—distribution center; goods-picking; conveyer belt

\section{INTRODUCTION}

The goods are mainly distributed to large-scale supermarkets and other wholesaling distribution centers originally, but now the business of distributing goods directly to individual customer grows rapidly along with the rapid development of BtoC e-commerce business, especially the emerging "cross-border e-commerce". By viewing its order data, this phenomenon indicates sharp increase in customer quantity and the expansion of order data scale. As a result, "goods-picking", which is one of the main businesses in distribution center, will cost more working hours, and the comprehensive operation cost of distribution center will increase.

Distribution centers often lead in conveyer belt for auxiliary goods-picking to reduce working hours. However, if the principle of reducing working hours by conveyer belt is not made clear, it will be impossible for distribution centers to determine whether conveyer belt lead-in is appropriate. In addition, lead-in and maintenance of conveyer belt need expenses, which will result in increase in comprehensive operation cost. Improper conveyer belt configuration even exists in "Hema Shengxian", the representative of "New Retailing" enterprises with strong technological team support [1].

*Fund Project: Special project of educational science "Thirteenth-five Year" Plan 2017 of Fujian Province "Ocean-Air Transit Center Design and Talent Cultivation" (Project No.: FJKYJD17-61).
With respect to the previous researches of distribution centers' goods-picking business and equipment selection, they can be generally divided into two categories. The first explains the logistics workflow using different theories, for instance, $\mathrm{Li}$ Yuzhou works out an optimization scheme of goods-picking and warehouse-out flow for a pharmaceutical company using VSA-WBS (value flow and work decomposition structure comprehensive analysis) [2]; Wu Nan obtains the optimal solution of the goods-picking scheme of a BtoC e-commerce warehouse by establishing the mixed integer programming model [3] and so on. Such achievements are conductive to the enhancement of people's understanding of logistics improvement from multiple perspectives. The second category focuses on providing guidance tools which can be rapidly applied to operation improvement. One of the representatives is the Distribution Center System written by Suzuki Shin who proposed EIQ analysis method [4]. The book stresses to grasp characteristics of distribution center by virtue of order data, and then selects the operation method and facilities and equipment according to the characteristics; similarly, Japan Institute of Logistics Systems also publishes Manual for Logistics Site Improvement at regular intervals [5]. In consideration of the general education background of the personnel engaged in logistics industry in China, such research achievements are relatively easy to be promoted in logistics industry. However, the above data is mainly the suggestion from an example, without explaining the theoretical principle and the decision-making step when selecting the equipment.

To sum up, this research aims to make clear the principle and effect of working hour reduction by using conveyer belt to pick goods, and to discuss the decision-making step of leading in conveyer belt for goods-picking. To this end, the model for calculating goods-picking working hour by order data is established in this research, and the difference in working hour constitution whether the conveyer belt is used for goodspicking when the scale of order data is different.

\section{DEFINITION AND EXPLANATION OF CORE CONCEPTS}

\section{A. Order Data}

The order data received by the distribution center consists of 3 basic projects, namely the order entry, item, and the ordered goods quantity, with relevant indexes shown as below. In this research, however, the increase in order entry or the ordered goods quantity is regarded as the increase of order data scale. 
E: Order Entry

I: Item

Q: Quantity

\section{L: Lines of Order-List}

IQ: Ordered Item Quantity

IK: Number of Customers Ordering Items

EQ: Quantity ordered by Customers

EN: Item ordered by Customers

\section{B. Goods-picking}

Goods-picking refers to a series of operations including picking the ordered commodities from warehouse of the distribution center till packaging and bundling of the time and delivering them out of warehouse.

\section{Use of Conveyer Belt for Goods-Picking}

When picking goods using conveyer belt, the box will be carried to various goods-picking zones by the conveyer belt, and the operator should pick out the commodity and put it into the box repeatedly. The operator will wait for the box during the process, so there may be waste of time, and distribution center will relieve this question through the following methods.

1) Setting temporary place for commodities: By setting temporary place for commodities in various goods-picking zones, the operator can put the picked items at temporary place if the box hasn't come, and then put all the commodities in when the box comes. There may be too many commodities needing to be put in simultaneously, so boxes of complex number will be made as a group and carried to one zone at the same time if this method is used as in "Fig. 1".

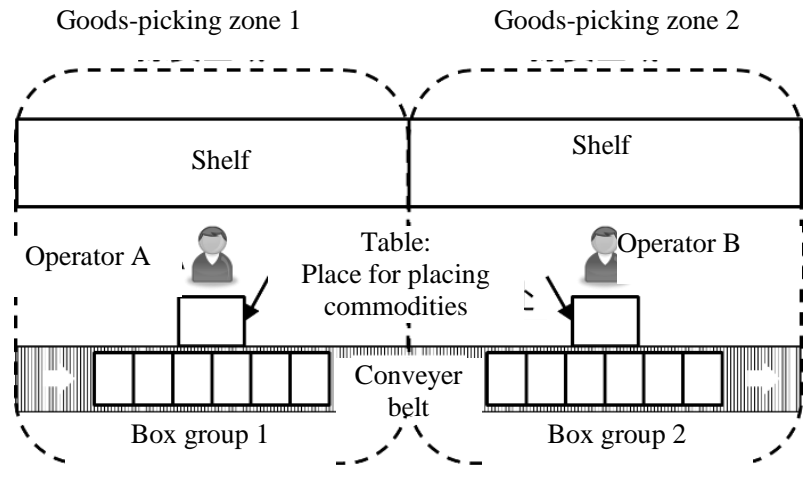

Fig. 1. Setting temporary place for commodities.

2) Setting a "pool" for boxes to be filled: Use the conveyer belts of complex number simultaneously; for instance, set 3 parallel conveyer belts, the middle one goes the opposite direction from the two side belts, and the goodspicking zones are set at the two sides of conveyer belt. When the boxes to be filled are conveyed by the middle belt, the operator should pick the box according to needs and put corresponding commodities in it, and then make them be carried away by the side belts. Such method is equivalent to setting a "pool" for boxes to be filled, and commodity putting will be completed at various goods-picking zones in a distributed way, which can reduce the time of waiting. In the implementation process, a set of methods for box recognition should be worked out reasonably as in "Fig. 2".

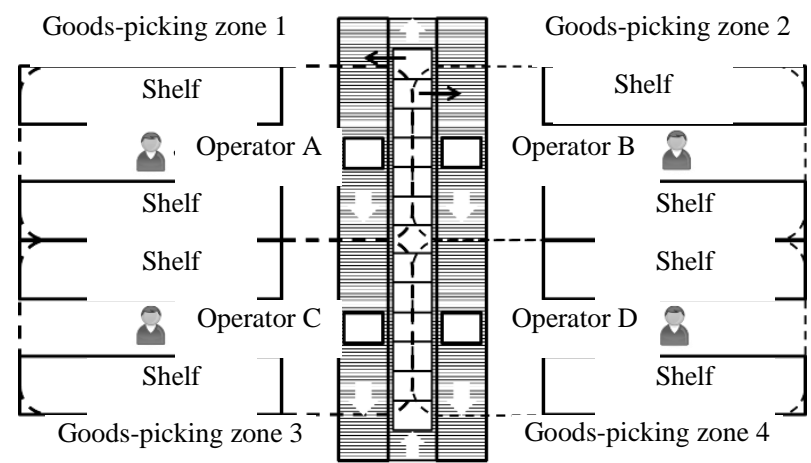

Fig. 2. Setting the "pool" for boxes to be filled.

3) Flow-line goods-picking: Set a conveyer belt with fixed speed, so that the boxes may flow to various goodspicking zones on the belt. The operator picks the commodity out from shelf and put it in corresponding box. This method is similar to the flow-line of factory, and the boxes here are like the products to be processed in the factory, goods-picking is like the operation of processing as in "Fig. 3". Goods-picking zone 1

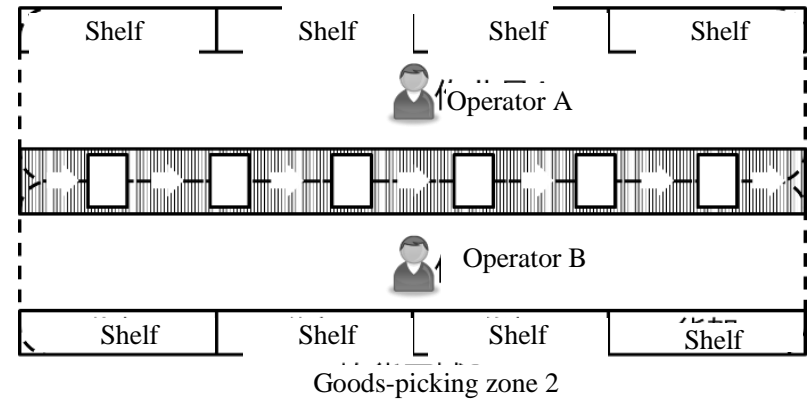

Fig. 3. Flow-line goods-picking.

4) Setting branch conveyer belt: Set branch conveyer belt at each goods-picking zone besides the main conveyer belt transporting the boxes. Only boxes needing to be filled with commodities in corresponding zone will be transported by branch conveyer belt, and other boxes will be conveyed to the next zone directly by the main conveyer belt. In this way, the boxes will not block the area where stopping is unnecessary, but in the process of implementation, classification of boxes according to goods-picking zones should be strengthened as in "Fig. 4". 


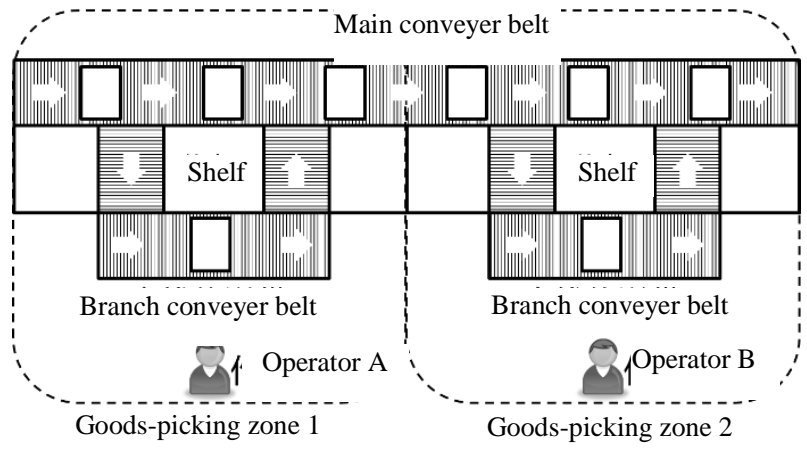

Fig. 4. Setting branch conveyer belt.

\section{Goods-Picking Time and Working Hour}

In this research, the goods-picking time is defined as the standard operation time of an operator to perform one time of goods-picking of commodities ordered by customers. When the conveyer belt is used, it will be the operation time at various goods-picking zones to perform one time of goodspicking. The sum of operation time is the working hour. Obviously, the working hour has a positive relation to the total salary the distribution center needs to pay to the operators.

\section{MODELING OF GOODS-PICKING WORKING HOUR}

\section{A. Content of Modeling}

In order to make clear the reason of working hour reduction of goods-picking by conveyer belt when there is large scale of order data, this research will establish the model for calculating goods-picking working hour using order data, including the working hour of goods-picking using conveyer belt, and the working hour of goods-picking using cart but not conveyer belt. The latter is used as a reference for comparison and analysis. Modeling of the working hour of goods-picking by cart will be performed first of all as its operation content is relatively basic, and then modeling of working hour of conveyer belt for goods-picking will be carried out.

Layout of goods-picking site set for modeling is shown in "Fig. 5". The left part of the figure is the site of goods-picking by cart, where the operator picks up commodities ordered by the customer in proper order using cart; the right part is the site of goods-picking by conveyer belt, the setting of which has referred to Method 1 and Method 2 for goods-picking by conveyer belt specified in Section 3 of Chapter 3. It is obvious that the layout of goods-picking site may change when the order scale changes. Therefore, "Fig. 5" shows the idea for setting goods-picking site layout while modeling; if more shelves are needed to keep the goods when there is large scale of order, the goods-picking site should be expanded according to this idea; and vice versa.

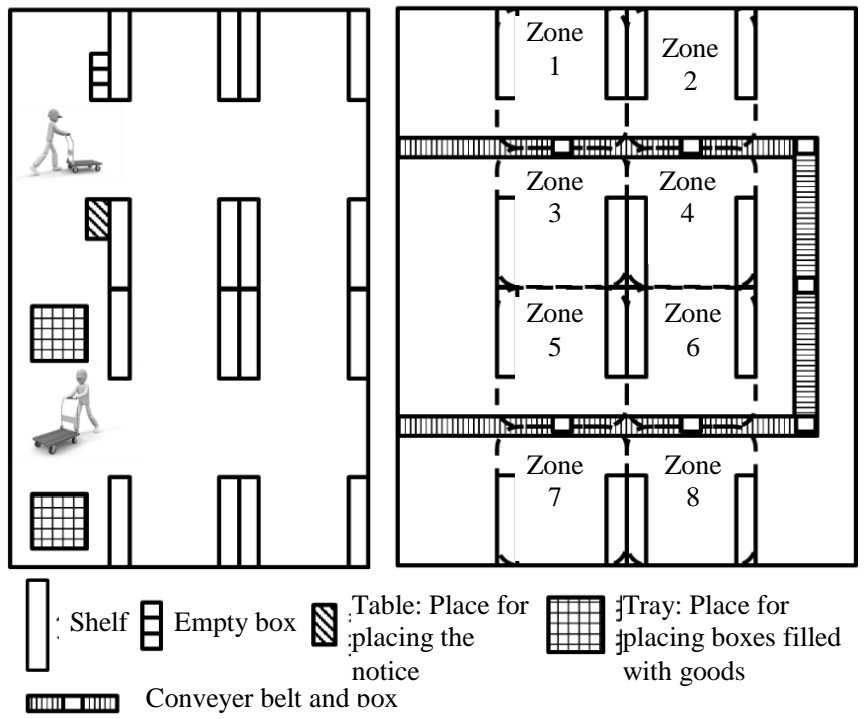

Fig. 5. Layout of goods-picking site by cart and conveyer belt.

\section{B. Modeling of Working Hour of Goods-picking by Cart}

The standard operation of goods-picking by cart includes 3 basic movements, namely "moving" to the shelf, "seeking" commodities, and "picking" commodities out. The working hour can be calculated by Formula (1) to Formula (4) by continuous use of the modeling method of the author's previous research [2].

$A T T_{\mathrm{i}}=G T T_{\mathrm{i}}+S T T_{\mathrm{i}}+M T T_{\mathrm{i}}$ Formula (1)

ATTi: Working hour of Customer i[s]

GTTi: Picking out (putting in) time of Customer i[s]

STTi: Seeking time of Customer i[s]

MTTi: Moving time of Customer i[s]

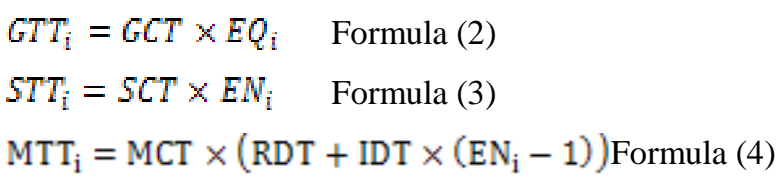

GCT: Coefficient of picking out [s/piece]

EQi: Ordered quantity of Customer i[piece]

SCT: Seeking coefficient [s/variety (line)]

ENi: Item of Customer i[variety]

MCT: Movement coefficient (reciprocal of movement speed) $[\mathrm{s} / \mathrm{m}]$

RDT: Average round-trip movement distance [m] [m]

IDT: Average movement distance between commodities

The sum of the above operation time is working hour, as shown in Formula (5). 


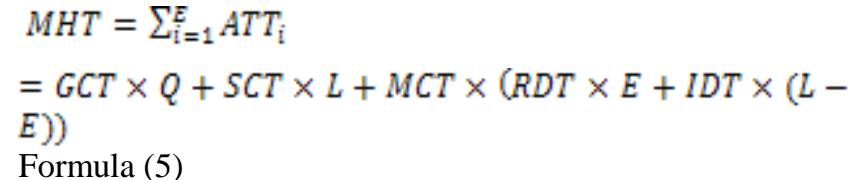

MHT: Working hour of goods-picking by cart [s]

\section{Modeling of Working Hour of Goods-Picking by Conveyer Belt}

When picking goods by conveyer belt, the operation flow in individual zone is shown in "Fig. 6". The operator should conduct a series of movements repeatedly as below: seek the storage position of commodity according to the notice of goods-picking, move to the storage position, pick out the commodity and put it into temporary container, and then move to the side of conveyer belt and put the commodity into box, press the button for confirmation on conveyer belt.

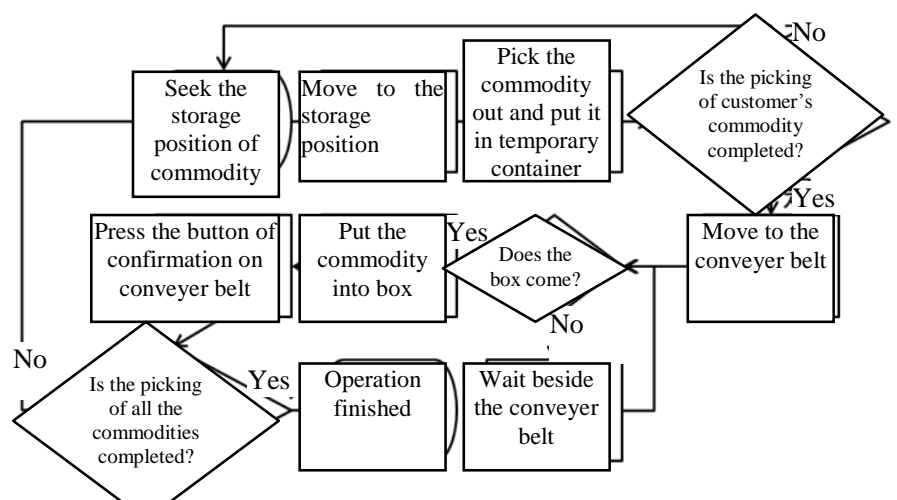

Fig. 6. Workflow in single zone of goods-picking by conveyer belt.

This series of actions is the same as that of goods-picking by cart, including 3 basic movements, namely "moving", "seeking" "picking out (putting in)". In addition, there will be the condition of "waiting" for the box transported by conveyer belt. First of all, the modeling will be conducted for the working hour after time of "waiting" is eliminated, namely "net working hour NTC", as show in Formula (6) to Formula (9).

$$
\begin{aligned}
& N T C_{j}=G T C_{j}+S T C_{j}+M T C_{\tilde{j}} \\
& =\sum_{i=1}^{E}\left(G T C_{i j}+S T C_{i j}+M T C_{i j}\right)
\end{aligned}
$$

Formula (6)

NTCj: Net working hour in Zone j [s]

GTCj: Picking out (putting in) time in Zone j [s]

STCj: Seeking time in Zone j [s]

MTCj: Moving time in Zone j [s] $\mathrm{j}[\mathrm{s}]$

GTCij: Picking out (putting in) time of Customer i in Zone

STCij: Seeking time of Customer i in Zone j [s]

MTCij: Moving time of Customer i in Zone j [s]

(7)

$$
G T C_{i j}=\left(G C C_{1}+G C C_{2}+G C C_{a}\right) \times E Q_{i j} \quad \text { Formula }
$$

$S T C_{i j}=S C C \times E N_{i j} \quad$ Formula (8)
(9)

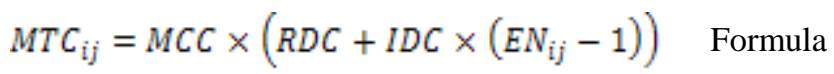

GCC1,GCC2,GCC3: Coefficient of picking out from (putting in) shelf, temporary container, box [s/piece]

EQij: Number of notes of Customer i in Zone j [piece]

SCC: Seeking coefficient [s/line]

ENij: Item of Customer i in Zone $\mathrm{j}$ [variety] $[\mathrm{s} / \mathrm{m}]$

MCC: Moving coefficient (reciprocal of movement speed)

RDC: Average round-trip moving distance $[\mathrm{m}]$

IDC: Average moving distance between commodities [m]

The working hour of goods-picking by conveyer belt is the sum of operation time of various zones, as shown in Formula (10).

$$
M H C=\Sigma_{j=1}^{Z} N T C_{j} \quad \text { Formula (10) }
$$

MHC: working hour of goods-picking by conveyer belt [s]

\section{Z: Zone number}

\section{COMPARISON OF GOODS-PICKING BY CART AND BY CONVEYER BELT}

\section{A. Discussion Content}

The changes in operation hour and relevant time of basic actions constituting working hour by each goods-picking method when order data scale occurs and how to choose ideal goods-picking method according to order data will be discussed in this part by comparing goods-picking by cart and by conveyer belt.

\section{B. Data Selection and Parameter Setting}

The order data and parameter used for calculation are shown in "Table I" and "Table II". Arrange the goods-picking site according the idea of "Fig. 6", set the width of shelf as $1.8 \mathrm{~m}$, distance between shelves as $1.8 \mathrm{~m}$, passage width as $2.0 \mathrm{~m}$, and then the coefficients will be obtained. In addition, some coefficients have referred to the setting in the author's previous research [6].

TABLE I. SUMMARY OF DATA FOR CLACULATION

\begin{tabular}{|l|l|l|l|l|}
\hline \multicolumn{2}{|c|}{ Elements } & \multirow{2}{*}{ Data 1 } & Data 2 & Data 3 \\
\cline { 1 - 2 } Mark & \multicolumn{1}{|c|}{ Description } & & 80 & 160 \\
\hline E & Order Entry & 40 & 500 & 2492 \\
\hline L & Item & 262 & 960 & 9600 \\
\hline Q & Lines of order-list & 480 & 960 & 9600 \\
& $\begin{array}{l}\text { Quantity of ordered } \\
\text { commodities }\end{array}$ & 480 & Small $\leftarrow \rightarrow$ Large \\
\hline Scale of order data & \multicolumn{2}{|l}{} \\
\hline
\end{tabular}


TABLE II. COEFFICIENT SET FOR CALCULATION

\begin{tabular}{|c|c|c|c|}
\hline Method & Mark & Coefficient Name [Unit] & $\begin{array}{c}\text { Value of } \\
\text { Coefficient }\end{array}$ \\
\hline \multirow{9}{*}{$\begin{array}{l}\text { Goods- } \\
\text { picking } \\
\text { by cart }\end{array}$} & GCT & $\begin{array}{l}\text { Coefficient of picking out } \\
\text { (putting in) (s/piece) }\end{array}$ & $2.200^{\text {note }}$ \\
\hline & SCT & Seeking coefficient [s/line] & $16.234^{\text {note }}$ \\
\hline & MCT & Moving coefficient [s/m] & $0.776^{\text {note }}$ \\
\hline & \multirow[t]{3}{*}{ RDT } & \multirow{3}{*}{$\begin{array}{l}\text { Average round-trip distance } \\
{[\mathrm{m}]}\end{array}$} & Data 1: 15.65 \\
\hline & & & Data 2: \\
\hline & & & Data 3: \\
\hline & \multirow[t]{3}{*}{ IDT } & \multirow{3}{*}{$\begin{array}{l}\text { Average moving distance } \\
\text { between commodities }[\mathrm{m}]\end{array}$} & Data 1: 6.58 \\
\hline & & & Data 2: \\
\hline & & & Data 3: \\
\hline \multirow{7}{*}{$\begin{array}{l}\text { Goods- } \\
\text { picking } \\
\text { by } \\
\text { conveyer } \\
\text { belt }\end{array}$} & GCC1 & \multirow{3}{*}{$\begin{array}{l}\text { Coefficient of picking out from } \\
\text { (putting in) shelf, temperary } \\
\text { container, box [s/piece] }\end{array}$} & $1.100^{\text {note }}$ \\
\hline & GCC2 & & $1.100^{\text {note }}$ \\
\hline & GCC3 & & $1.100^{\text {note }}$ \\
\hline & SCC & Seeking coefficient [s/piece] & $16.234^{\text {note }}$ \\
\hline & $\mathrm{MCC}$ & Moving coefficient $[\mathrm{s} / \mathrm{m}]$ & $0.776^{\text {note }}$ \\
\hline & RDC & $\begin{array}{l}\text { Average round-trip distance } \\
{[\mathrm{m}]}\end{array}$ & 2.776 \\
\hline & IDC & $\begin{array}{l}\text { Average moving distance } \\
\text { between commodities }[\mathrm{m}]\end{array}$ & 1.573 \\
\hline
\end{tabular}

a. Note: The setting has referred to Reference [6]

\section{Influence of Order Data Scale}

1) Goods-picking by cart: The working hour of goodspicking by cart and its constitution are shown in "Fig. 7". It can be known from the figure that, working hour increases along with the increase in order data scale, and the ratio of moving time goes up sharply from $24 \%$ to $43 \%$.

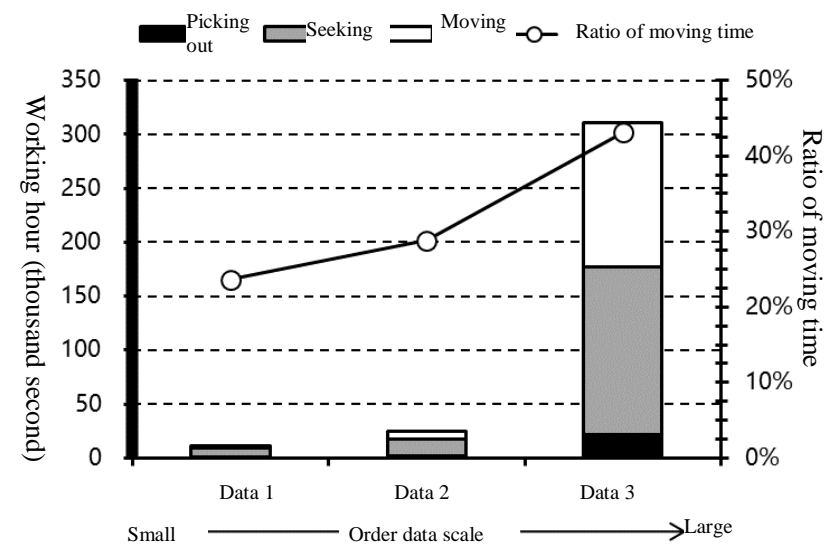

Fig. 7. Working hour of goods-picking by cart and its constitution.

2) Goods-picking by conveyer belt: The working hour of goods-picking by conveyer belt and its constitution are shown in "Fig. 8". It can be known from the figure that, the working hour also increases along with the increase in order data scale, but the ratio between moving time and net working hour remains almost unchanged $(8 \%-10 \%)$.

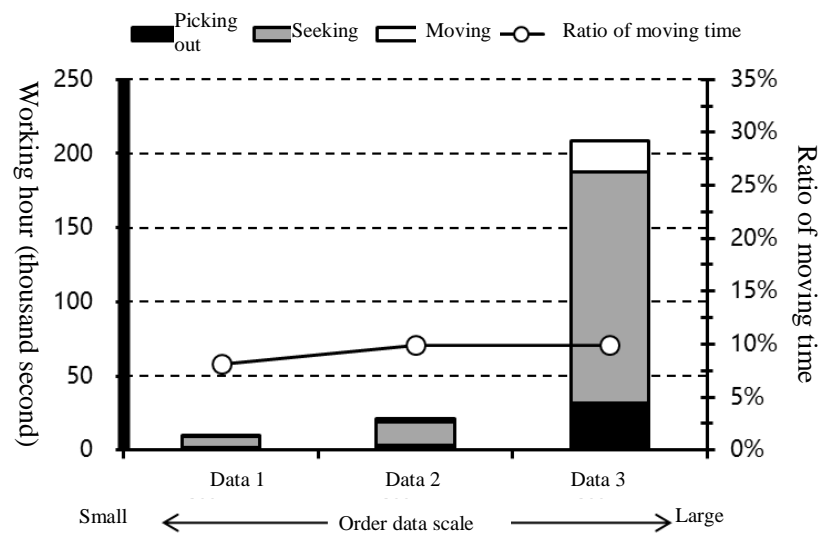

Fig. 8. Working hour of goods-picking by conveyer belt and its constitution.

\section{Comparison of Working Hour}

The comparison of working hour of two goods-picking methods is shown in "Fig. 9". It can be known from the figure that, when order data scale is small, there is no big difference in the working hour of two goods-picking methods, even the working hour without conveyer belt is shorter; but when there is large order data scale, the working hour of goods-picking by conveyer belt will be obviously shorter than that of cart. Therefore, the distribution center can reduce the working hour effectively by using conveyer belt for goods-picking when there is large order data scale.

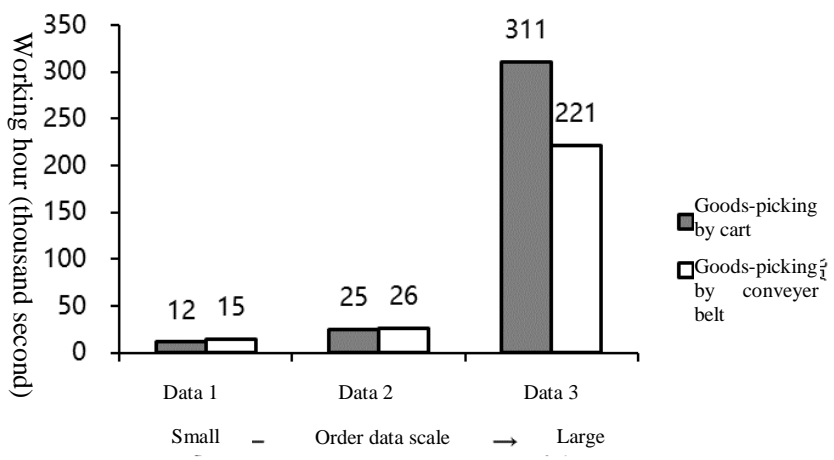

Fig. 9. Comparison of working hour of two goods-picking methods.

In addition, the comparison of the ratio of moving time in working hour of the two goods-picking methods is shown in "Fig. 10". It can be seen from the figure that, the moving time ratio of goods-picking by cart is always larger than that by conveyer belt, and the difference value increases along with the increase of order data scale. It can be considered that, the reason why goods-picking by conveyer belt can reduce working hour is that it can reduce operator's moving time. 


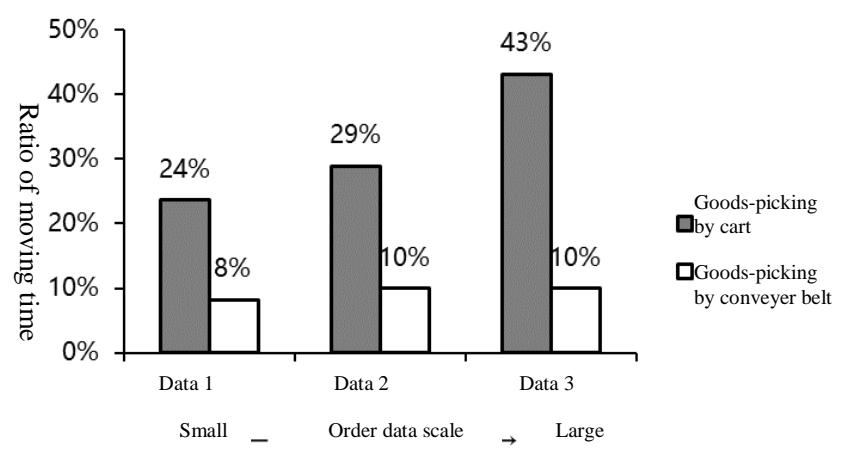

Fig. 10. Comparison of moving time ratio of two goods-picking methods.

To further explore the reason of reduction of operator's moving time by conveyer belt for goods-picking, the routes of operator in goods-picking by cart and by conveyer belt are drawn respectively, as shown in "Fig. 11". It can be seen from the figure that, the walking distance of each operator will increase along with the expansion of goods-picking site when picking goods using cart; but if the conveyer belt is used for goods-picking, the size of each goods-picking zone will remain unchanged as the expansion of goods-picking site is the increase in goods-picking zones, so the walking distance of each operator will not increase. Using conveyer belt for goodspicking is appropriate when the order data scale is large as the size of goods-picking site of distribution center has positive relation with order data scale.

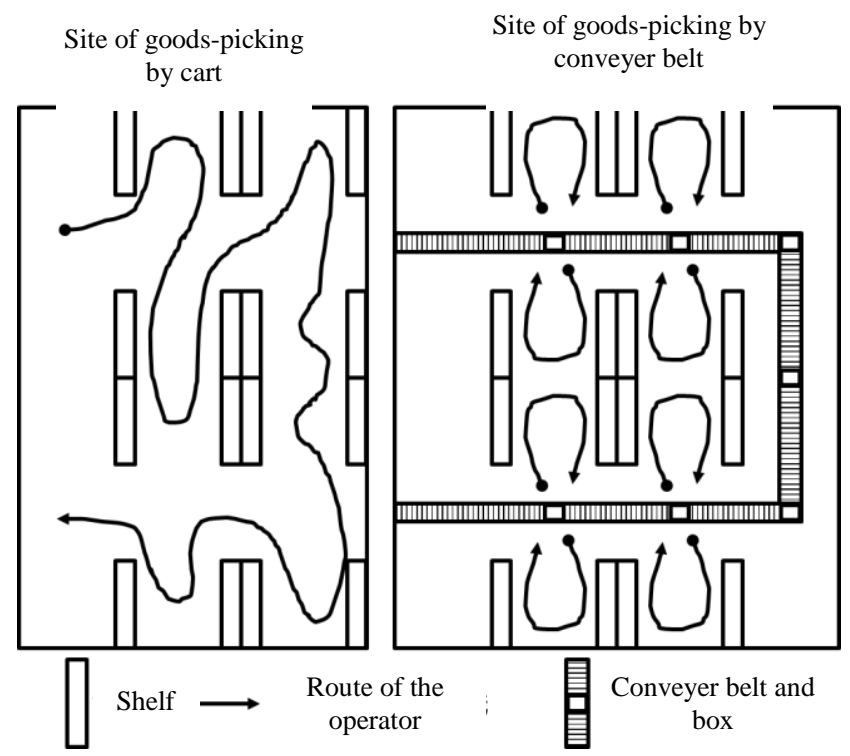

Fig. 11. Route of goods-picking operator.

\section{E. Steps for Determining Selection of Conveyer Belt for Goods-picking}

When determining whether the conveyer belt should be used for goods-picking, the distribution center can take comprehensive operation cost as the judgment index, with specific steps for judgment as shown below.
First of all, calculate the working hour of goods-picking with and without conveyer belt by referring to the calculation model of working hour of goods-picking by cart and by conveyer belt in this research, and the data and parameter to be collected during the calculation process can refer to Table 5-1 and "Table II". Of course, the specific value should be based on the order data and the condition of facilities and equipment of various distribution centers.

After the calculation value is obtained, whether conveyer belt can reduce the working hour of goods-picking can be determined. If the answer is Yes, multiply the reduced working hour by average salary of unit working hour, and then the salary cost which can be reduced is obtained, as shown in Formula (11).

$$
L C D=L \times(\mathrm{MHT}-\mathrm{MHC}) \quad \text { Formula (11) }
$$

\section{LCD: Reduced salary cost}

\section{L: Average salary of unit working hour}

\section{MHT: Working hour of goods-picking by cart}

\section{MHC: Working hour of goods-picking by conveyer belt}

If the reduced salary cost is larger than the cost of conveyer belt lead-in and maintenance (time unit needs to be unified in the process of comparison), then the using of conveyer belt will reduce the comprehensive operation cost.

As for the distribution center that hasn't used conveyer belt or hasn't used it in an all-round way, its order data scale will increase gradually with the development of business, meaning that the reduced working hour by conveyer belt lead-in will also increase in "Fig 12". Therefore, the comprehensive operation cost will be reduced by conveyer belt lead-in at appropriate time if decision and judgment are made in a planned way at regular intervals.

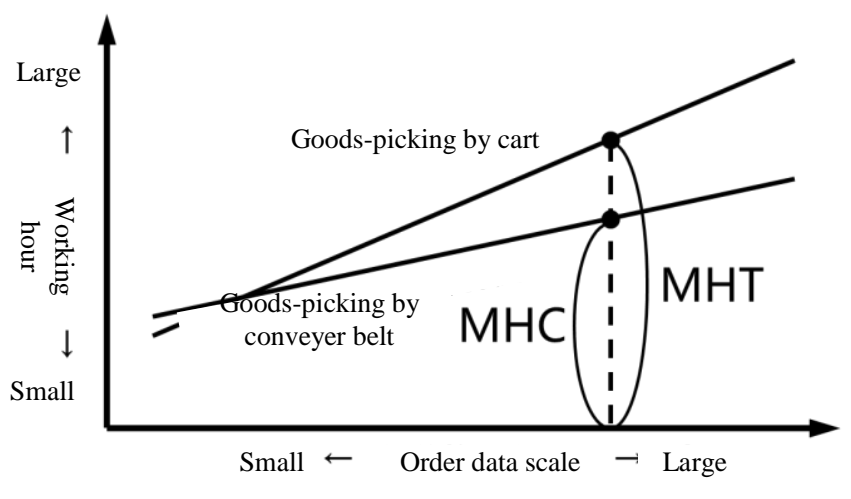

Fig. 12. Effect of working hour reduction by conveyer belt for goods-picking.

\section{CONCLUSION}

In this research, the goods-picking operation at distribution center is taken as discussion object, modeling, calculation and comparison of the working hour of goods-picking with and without conveyer belt under different order data scales are performed, and the following conclusion is obtained. 
First, the moving time of each operator will increase along with the increase of order data scale if conveyer belt is not used for goods-picking, which condition will be avoided if conveyer belt is used. Therefore, it is appropriate to use conveyer belt for goods-picking in distribution center with large order data scale.

Second, distribution center can determine whether the conveyer belt should be led in for goods-picking by judging whether the comprehensive logistics cost is reduced, and such judgment and decision should be carried out in a planned way at regular intervals to realize the reduction of comprehensive logistics cost.

However, the cost of conveyer belt lead-in and maintenance in distribution center is not discussed in this research; moreover, the possible waiting time in goods-picking by conveyer belt is not taken into full consideration. As a result, these problems will be discussed in the future.

\section{REFERENCES}

[1] Wang Xiaoran, Xu Tianyue. Is the Conveyer Belt of "HeMa" Still Running [N]. Beijing Business Today, 2018-6-13, Ver. C01: 1-2.

[2] Li Yuzhou. Study on DLK Module Warehouse-out Flow Optimization based on Value Flow Analysis [D]. Yunnan University of Finance and Economics, 2018.

[3] Wu Nan. Study on Order Selection Operation Optimization of A Ecommerce Enterprise Warehouse Center [D]. Jinan University, 2016.

[4] Suzuki Shin. Distribution Center System [M]. Tokyo: Seizando-Shoten Publishing Co., Ltd., 1997:8-14.

[5] Manual for Logistics Site Improvement [M]. Tokyo: Japan Institute of Logistics Systems, 2018:15-29.

[6] Ke Shengjie. Research on Goods-picking Method Selection based on Order Data [J]. Journal of Hubei Polytechnic University (Humanities and Social Sciences), 2017(6): 33-37+42. 